\title{
The Perception of Fluctuations of Cell Velocity as a Possible Means of Overcoming Sedimentation by Motile Sea-Plankton Microorganisms
}

\author{
Pundyak Oleh* \\ Botanical Garden of Ukrainian National Forestry University, Ukraine \\ *Corresponding author: Pundyak Oleh, Botanical Garden of Ukrainian National Forestry University, Ukraine
}

\begin{abstract}
A model for overcoming the gravity by cells of wide range of motile sea-plankton microorganisms without gravity perception or antipredator behavior is proposed here. It is based on the assuming that the cells are able to detect thermal fluctuations of their velocities. In our model the cell locomotion force linearly depends on the cell velocity fluctuations causing especial type of gravikinesis. According to equations of the cell movement, sedimentation of the organisms may be considerably overcome.
\end{abstract}

Keywords: Microplankton; Picoplankton; Sedimentation; Gravikinesis

\section{Introduction}

The speed of some microorganisms depends on their orientation within the gravity field (excluding buoyancy of the whole cells). This phenomenon is called gravikinesis. Physiological mechanisms of gravikinesis have been well examined and described by Hemmersbach \& Häder et al, [1] for example. It is expressed by the existence of certain cell receptors accepting gravistimulus and, through signaling chains, causing the change of cell motor work. The physical aspect of gravikinesis might be expressed by an anisotropic interaction of the microorganisms with the water medium. Recent calculations [2] supported our hypothesis that the physical aspect of gravikinesis can occur in bacterial and also in eukaryotic motile sea-picoplankton cells. According to our mentioned calculations the physical aspect of gravikinesis can be visible only in the case of high concentrations of bacteria without antipredator behavior (AB) equaling or bigger than $10^{8}$ cells $\mathrm{m}^{-3}$ in consequence of friction anisotropy of co-existing microorganisms with high level of $\mathrm{AB}$. Thus, a question can be raised: whether motile sea-plankton microorganisms in low concentrations can overcome gravity without any special cellular mechanism for gravity perception, as it has been shown for asymmetric microplankton $[3,4]$ or for sea-picoplankton with antipredator behavior [2] ? We have tried to answer this question positively taking into account the existing of mechanosensitive channels, which can detect the changes of cell membrane tension linked with the changes of friction force during fluctuations of cell velocity [6]. Let us show the possible result when an averaged fluctuation force, which acts on the cells with ability to detect the thermal fluctuations of their velocities, is considerable in comparison with buoyance force. As the velocity fluctuations are very ephemeral (their duration for plankton organisms with dimensions up to $10^{-5} \mathrm{~m}$ does not exceed $10^{-5} \mathrm{~s}$ ), they can be detected as the difference between the real cell velocities and averaged ones:

$$
\Delta \dot{x}^{\prime}(t) \equiv \dot{x}^{\prime}(t)-\overline{\dot{x}^{\prime}}(t) ; \Delta \dot{y}^{\prime}(t) \equiv \dot{y}^{\prime}(t)-\overline{\dot{y}}(t) ; \Delta \dot{z}^{\prime}(t) \equiv \dot{z}^{\prime}(t)-\overline{\dot{z}}(t)
$$

where $\Delta \dot{x}^{\prime}, \Delta \dot{y}^{\prime}, \Delta \dot{z}^{\prime}$ are the cell velocity fluctuations. Let us consider such a velocity fluctuations detection in our model system. For this goal let us transform equations (1), (2), (3) [2] having considered equation (9) [2]. Thus, analogically to equation (11) [2] we obtain: 


$$
\begin{aligned}
& m \ddot{x}^{\prime}+\left(h_{x^{\prime}}+\beta\right) \cdot \Delta \dot{x}^{\prime}=A_{x^{\prime}}(t), m \ddot{y}^{\prime}+h_{y^{\prime}} \Delta \dot{y}^{\prime}=A_{y^{\prime}}(t) \\
& m \ddot{z}^{\prime}+h_{z^{\prime}} \Delta \dot{z}^{\prime}=A_{z^{\prime}}(t)
\end{aligned}
$$

where $\beta$ is the coefficient of the cell velocity fluctuations detection. If $\beta=$ const, then it does not influence on the sedimentation rate. But it is natural to consider that the receptors of the velocity fluctuations detect only scalar velocity deviations. So, in our model $|\beta|=$ const and when $\Delta \dot{x}^{\prime}$ change its sign, $\beta$ does it too. There appear two variants: with positive and negative values of $\beta \cdot \Delta \dot{x}^{\prime}$. The first corresponds to the inhibition of the cell motor as the respond on the deviations. The latter - to the stimulation. Thus, according to [2] during the averaging of equation (2) for $\Delta \dot{x}^{\prime}$ , averaged fluctuation force (let us call it diffusional force) may be not equal zero:

$$
\Lambda \equiv \bar{A}_{x^{\prime}}=\beta \cdot \Delta \overline{\dot{x}}^{\prime}
$$

Thus, formula (18) [2] changes as

$$
\begin{aligned}
& \stackrel{\Lambda}{E}=X(t) \frac{D_{x}}{d_{x}} \beta=X(t) \frac{k_{b} T}{d_{x}} \xi ; \xi \equiv \frac{\beta}{h_{x}^{\prime}} \\
& \chi(t) \approx \frac{d_{x^{\prime}} d_{y^{\prime}, z^{\prime}}^{2}}{\left(B_{x^{\prime}} t^{0,5}+d_{x^{\prime}}\right)\left(B_{y^{\prime}, z^{\prime}} t^{0,5}+d_{y^{\prime} z^{\prime}}\right)^{2}} ; B_{x^{\prime}} \equiv \sqrt{D_{x^{\prime}}} ; B_{y^{\prime}, z^{\prime}} \equiv \sqrt{D_{y^{\prime}, z^{\prime}}} \quad \text { where }
\end{aligned}
$$
$\mathrm{d}_{X^{\prime}}$ and $\mathrm{d}_{y^{\prime}, z^{\prime}}$ are the dimensions of the particles along the axes $\mathbf{X}^{\prime}$ and $\mathbf{Y}^{\prime}$ or $\mathbf{Z}^{\prime}$ (of orthogonal coordinate system bounded with the axis X', along which the locomotion force always acts) correspondingly; $\mathrm{D}_{x^{*}}$ and $\mathrm{D}_{y^{\prime}, z^{\prime}}$ are coefficients of diffusion along the axis $\mathbf{X}^{\prime}$ and $\mathbf{Y}^{\prime}$ or $\mathbf{Z}^{\prime}$ correspondingly. It is natural to assume that $\beta \geq \mathrm{h}_{x^{\prime}}$. Let us consider that

$$
\xi \approx 10
$$

Thus, according to all calculations done in [2] not only for picoplanktonic (as it was shown for the cells with antipredator behavior) but for a part of microplanktonic organisms (with the dimensions from $2 \cdot 10^{-6} \mathrm{~m}$ up to $8 \cdot 10^{-6} \mathrm{~m}$ ) the diffusional force may have a considerable value in comparison with buoyant force. Thus, we can say, that picoplanktonic organisms and the part of microplanktonic ones with their dimensions up to $8 \cdot 10^{-6} \mathrm{~m}$, which have the cell velocity fluctuations receptors can considerably decrease sedimentation without any special receptors of gravity. We also can conclude that, though poorly studied generally and especially in the world of motile picoplankton and microplankton, the receptors of cell velocity fluctuations can also serve as an adaptation to overcome sedimentation. This finding may stimulate further research in this field.

\section{References}

1. Hemmersbach R, Häder, DP (1999) Graviresponses of certain ciliates and flagellates. FASEB J 13: 69-75.

2. Pundyak $O$ (2017) Possible means of overcoming sedimentation by motile sea-picoplankton cells. Oceanologia 59 (2): 108-112.

3. Hagen B, Kümmel F, Wittkowski R, Takagi D, Löwen H, et al. (2014) Gravitaxis of asymmetric self-propelled colloidal particles. Nat Comm 5(9): $1-7$

4. Roberts AM, Deacon FM (2002) Gravitaxis in motile micro-organisms: The role of fore-aft body asymmetry. J Fluid Mech 452(1): 405-423.

5. Rasmussen $\mathrm{T}$ (2016) How do mechanosensitive channels sense membrane tension? Biochem Soc Trans 44(4): 1019-1025.

\section{(C) This work is licensed under Creative}

To Submit Your Article Click Here: Submit Article

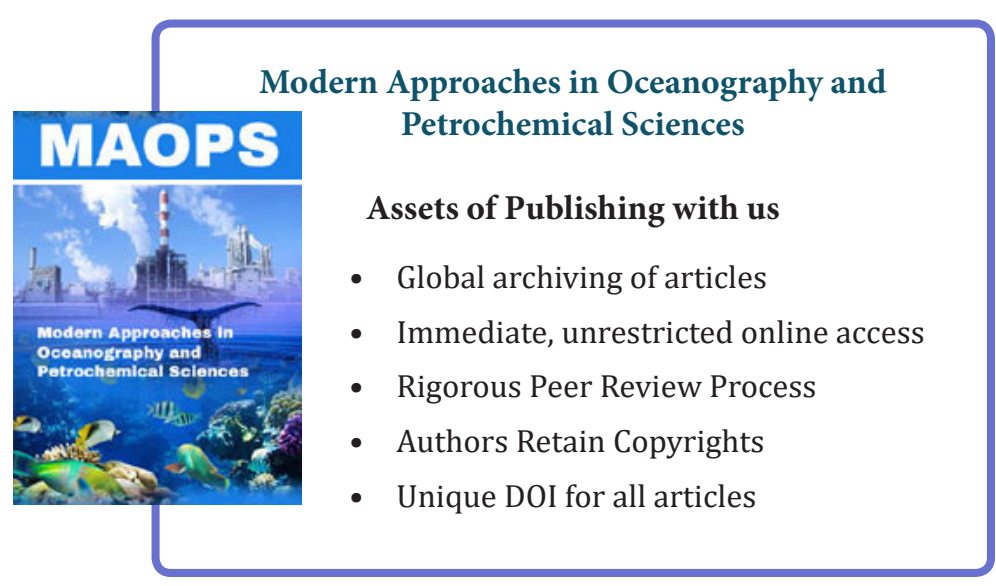

